ERRATUM OPEN

\title{
Erratum: Electroconvulsive therapy-induced brain functional connectivity predicts therapeutic efficacy in patients with schizophrenia: a multivariate pattern recognition study
}

Peng $\mathrm{Li}^{1}$, Ri-xing Jing ${ }^{2,3}$, Rong-jiang Zhao ${ }^{4}$, Zeng-bo Ding ${ }^{5}$, Le Shi ${ }^{1,5}$, Hong-qiang Sun ${ }^{1}$, Xiao Lin ${ }^{6}$, Teng-teng Fan ${ }^{1}$, Wen-tian Dong ${ }^{1}$, Yong Fan $^{7}$ and Lin $\mathrm{Lu}^{1,5,6}$

npj Schizophrenia (2017)3:33; doi:10.1038/s41537-017-0024-6

Erratum to: npj Schizophrenia (2017); doi:10.1038/s41537-0170023-7; Published 11 May 2017

The Competing interests section states, "The authors declare competing financial interests." However, it should be, "The authors declare no competing financial interests." These errors have now been corrected in the HTML and PDF versions of this article.

Open Access This article is licensed under a Creative Commons Attribution 4.0 International License, which permits use, sharing, adaptation, distribution and reproduction in any medium or format, as long as you give appropriate credit to the original author(s) and the source, provide a link to the Creative Commons license, and indicate if changes were made. The images or other third party material in this article are included in the article's Creative Commons license, unless indicated otherwise in a credit line to the material. If material is not included in the article's Creative Commons license and your intended use is not permitted by statutory regulation or exceeds the permitted use, you will need to obtain permission directly from the copyright holder. To view a copy of this license, visit http://creativecommons. org/licenses/by/4.0/.

(c) The Author(s) 2017

\footnotetext{
${ }^{1}$ Institute of Mental Health, National Clinical Research Center for Mental Disorders, Key Laboratory of Mental Health and Peking University Sixth Hospital, Peking University, Beijing 100191, China; ${ }^{2}$ National Laboratory of Pattern Recognition, Institute of Automation, Chinese Academy of Sciences, Beijing 100190 , China; ${ }^{3}$ University of Chinese Academy of Sciences, Beijing 100049, China; ${ }^{4}$ Department of Alcohol and Drug Dependence, Beijing Hui-Long-Guan Hospital, Peking University, Beijing 100096, China; ${ }^{5}$ National Institute on Drug Dependence and Beijing Key laboratory of Drug Dependence, Peking University, Beijing 100191, China; ${ }^{6}$ Peking-Tsinghua Center for Life Sciences and PKU-IDG/ McGovern Institute for Brain Research, Peking University, Beijing 100871, China and ${ }^{7}$ Department of Radiology, Perelman School of Medicine, University of Pennsylvania, Philadelphia, PA 19104, USA
}

Correspondence: Yong Fan (yong.fan@ieee.org) or Lin Lu (linlu@bjmu.edu.cn)

Peng Li and Ri-xing Jing have contributed equally to this work. 